\title{
First results from a pre-operational system for automatic detection and recognition of seismic signals associated with avalanches
}

\author{
Benoit J. P. Leprettre, Jean-Pierre Navarre and Alain Taillefer \\ Centre d'Étude de la Neige, Météo-France, 38406 Saint-Martin-d'Hères, France
}

\begin{abstract}
A system for automatic, reliable, semi-instantaneous estimation of avalanche activity is presented in an attempt to check deterministic models and improve the surveillance of risk zones. The principle is the seismic detection of avalanches. Two experimental sites equipped with standard seismological equipment are described. Avalanche seismic signals are recorded, as well as many extraneous signals of natural or human origin. After several years of a posteriori identification of the signals, we are now able to set up criteria for the automatic recognition of nonavalanche signals. We have therefore developed an automatic analysis/decision system to discriminate between avalanche signals and others. This system worked satisfactorily in pre-operational conditions during the winter of 1995 - 96 . The results are presented and compared with other data related to avalanche activity. Although there is still room for improvement, our system seems to be able to estimate avalanche activity better than human visual observations.
\end{abstract}

\section{INTRODUCTION}

According to studies carried out by the French Association Nationale pour l'Étude de la Neige et des Avalanches (ANENA) - National Snow and Avalanche Research Association - for the 1971-90 period, avalanches are responsible for an average of 36 serious accidents in France each year, causing 28 dead and 26 injured (Valla and Tuaillon, 1991). About $20 \%$ of these avalanches are natural avalanches, i.e. avalanches that have not been triggered by an alpinist or a skier. Famous natural avalanches are well remembered: 39 people were killed in Val d'Isère, France, on 10 February 1970. Since then, the properties of snow and avalanches have been investigated at the Centre d'Étude de la Neige (CEN) - Snow Research Centre, a department of Météo-France, the French Meteorological Office, in order to attempt to understand the avalanche phenomenon better.

At present, an estimate of the natural activity of a massif is derived from human visual observations. As no visual observations are possible at night, or when the weather is cloudy, there is a time bias in the estimation of avalanche activity. Furthermore, deposits of airbornepowder avalanches are sometimes almost invisible (especially if another layer of fresh snow settles on the deposits before the weather clears) and cannot always be observed afterwards, even when the weather has cleared. Thus, there is a second bias concerning the intensity of an avalanche episode. Consequently, the evolution versus time of avalanche activity during and after a snowfall is not well known. Nevertheless, reliable information on this point would be very useful. It would allow us to check the deterministic model for estimation of the avalanche hazard (the SAFRAN-CROCUS-MEPRA chain) developed by the CEN (Giraud and others, 1994). From a more practical point of view, a real-time estimate of avalanche activity would help in taking decisions concerning closing or re-opening of roads or ski tracks threatened by avalanches. It would also help meteorologists to forecast the short-term avalanche hazard, given the current avalanche activity and weather conditions.

To solve this problem, a research team at CEN is investigating the possibility of detecting avalanches in real time by using seismic methods. The aim is to produce a reliable real-time estimate of the avalanche activity of a massif, whatever the weather conditions. The principle of seismic detection of avalanches (SDA) is simple. When an avalanche occurs, seismic waves are generated into the ground by descending snow "packets". These waves can be detected by seismic sensors installed in the vicinity of the avalanche path. It is therefore possible to estimate the avalanche activity in a given area by observing its seismic activity. Previous studies (St. Lawrence and Williams, 1976; Navarre and others, 1991) have proved the feasibility of the seismic detection of avalanches. Other SDA experiments are being conducted in the Spanish Pyrenees (Sabot and others, 1995). However, a major problem has arisen: the seismic station records avalanche signals as well as many extraneous signals, such as earthquakes, blasts, footsteps and truck, helicopter or thunder sounds. It is therefore important to distinguish avalanche signals from other types of signal. In order to do this, after a trial period when the signals were identified using later information, an automatic recog- 
nition system based on a time-frequency-polarization analysis of the signal was developed. Fuzzy logic is used to take account of the empirical nature of our knowledge about avalanche recognition.

In section 1, the instruments and the two equipped sites are described, as well as the methodology for the a posteriori identification of the signals. Section 2 briefly presents the fuzzy-logic expert system we have developed for the automatic recognition of the signals. Finally, experimental results are presented in section 3 and this is followed by concluding remarks.

\section{SEISMIC DETECTION OF THE AVALANCHES EXPERIMENT AT CEN}

In this section, the instruments and the two experimental sites are described. Following this, the methodology for the a posteriori identification of signals during the training phase is explained.

\subsection{Instruments and sites}

The seismological stations used for seismic detection of avalanches (SDA) are basically composed of three main parts: the sensor, the seismological station and the communication system (Fig. 1). The sensor is a threecomponent Mark Products geophone. It is linear in the 2 $40 \mathrm{~Hz}$ frequency band, in which avalanche signals are located. The station itself consists of a detection device and storage hardware. The detection of seismic signals is performed by a permanent short-time average/long-time average (Sta/Lta) calculation with a threshold test. When a signal is detected, it is recorded on a hard disk with given pre- and post-events. The sampling frequency is $100 \mathrm{~Hz}$. The file name of the event contains the time and date at triggering. Compared with a standard seismological $\log$ used for earthquake detection, two slight modifications are needed for avalanche detection. First, the duration of the pre-event, i.e. the part of the signal

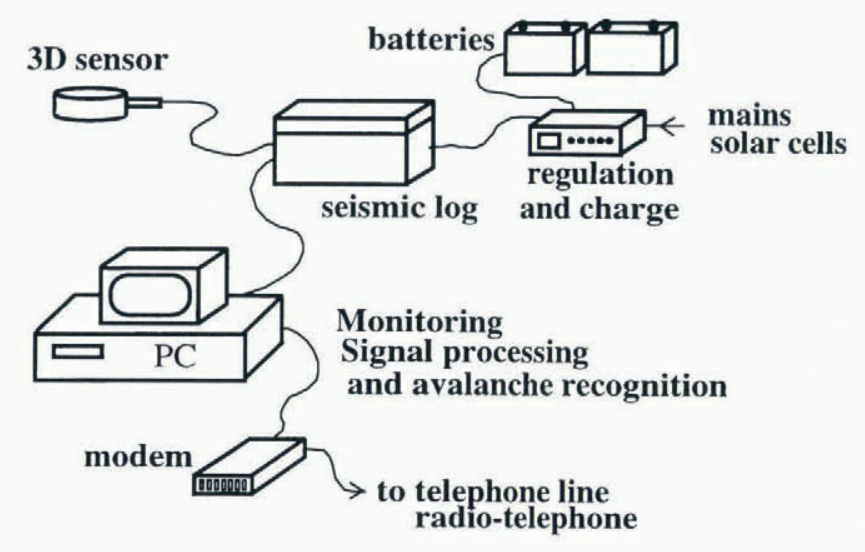

Fig. 1. Basic instrumentation used for the seismic detection of avalanches experiment. The sensor is a three-component Mark Products geophone, the detection and storage device is a CEIS three-component seismological station. The power is supplied via a regulation system by the mains (on the Saint-Christophe site) or solar cells (on the La Lavey site). prior to detection that will be recorded on the disk, must be much longer to avoid cutting short the beginning of the avalanche signals, the energy of which increases in general at a slower and smoother rate. The duration of the post-event must also be longer. Secondly, the detection threshold must be lower, in order to detect low-amplitude avalanches. The communication system consists of a modem, allowing remote monitoring of the seismic station. Once connected, one can modify the detection parameters (the length of Sta or Lta, threshold value of Sta/Lta) or the recording parameters (pre- or post-event length). It is also possible to determine how many signals have been recorded and to transfer signals to the laboratory for further analysis.

Two sites have been instrumented since 1993: the La Lavey site and the Saint-Christophe site. Each has its own characteristics. The La Lavey site is located at the mountain refuge of La Lavey, Oisans Massif, French Alps, at an altitude of $1800 \mathrm{~m}$. The seismic station is inside the refuge but the sensor is $20 \mathrm{~m}$ away, outside the refuge. The power supply is two $12 \mathrm{~V}$ batteries connected to solar cells placed on the roof of the refuge. Communication between the seismic station and the Snow Research Centre is by a modem and the radiotelephone line to the refuge. The site is at the bottom of a small, sheltered north-south valley, with no road nearby and surrounded by steep slopes, some of which are covered by glaciers or seracs. The altitude of the highest summits along the valley is $3600 \mathrm{~m}$, so that large avalanches are likely to occur. This site, although prone to avalanches, has a major drawback: it is not completely visible from the nearest road and several large avalanche corridors have to be crossed to have access to it. Therefore, we cannot reach the site on foot and make observations when the risk of avalanches is high. Neither can we have access to the site by air, because it is within the National Park of Les Ecrins, above which the use of helicopters is strictly restricted. In spite of this lack of avalanche observation, this site extends our collection of signals of all kinds, thus expanding our data base. It is also much used to cross-check the date and time information from other stations and therefore to spot earthquake signals: if a signal of a similar amplitude is detected at the same time by two stations a few kilometres apart, then the corresponding event is almost certain to be an earthquake.

The Saint-Christophe site, on the other hand, is located on the slope of a valley, at an altitude of $1700 \mathrm{~m}$ and not far from a small mountain road. Access is therefore easier than for La Lavey. The distance between the two sites is $5 \mathrm{~km}$. The terrain around the station is a steep, even slope facing southwest with scree and grass. The instrumentation is a seismic sensor located on scree, $400 \mathrm{~m}$ above the road. The seismic station is $15 \mathrm{~m}$ away from the sensor and in an insulated "case". The secondary equipment is more complete than at $\mathrm{La}$ Lavey. A cabin near the road contains a PC computer, a modem and a Campbell weather station. The power for all the equipment, including the seismic log, is from the mains using regulating devices. However, the seismic log is also connected to two $12 \mathrm{~V}$ batteries, in case of mains power failure. The seismic log and the Campbell station are monitored by the computer. The Campbell station provides measurements of wind speed and air temper- 
ature. These data have allowed us to check that wind squalls have not produced extraneous signals, provided that the sensor is protected by a concrete box. This modem also allows remote logging on the computer from the Snow Research Centre. The triggering and recording parameters of the seismic log can then be adjusted, interesting signals can be transferred from the station to the computer and from there to the laboratory if necessary. Since December 1994, the computer has also been responsible for analysing the recorded signals and deciding whether a given signal has been produced by an avalanche or not. This task is actually the key point in an operational context and will be described further in the paper. Since January 1995, four well-known avalanche gullies close to the seismic station have been equipped with avalanche detectors. These consist of simple batterypowered watches with date indicators installed inside waterproof cases beside the avalanche corridors (Fig. 2). A plug is connected in series with the battery and is linked to a set of stakes set in the snow in the avalanche gully. When an avalanche occurs in one of these equipped gullies, the stakes are broken, the plug is ejected and the watch stops. It is simply necessary to open the case later to know exactly when the avalanche occurred. New stakes can then be set up and the whole device is once again operational. This simple and cheap system works remarkably well and has allowed us to identify some of the avalanche signals.

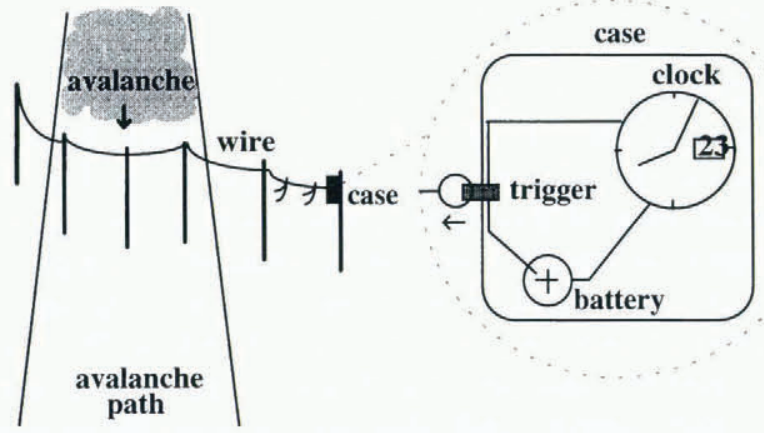

Fig. 2. Principles of a simple and efficient avalanche detector and date indicator. The avalanche destroys the stakes and the plug connected in series with the battery is ejected and the watch stops, showing the date and time when the avalanche occurred.

To summarize, the two sites have different aims. The La Lavey site, because of its isolation, is principally used to test the behaviour of the equipment in the severe winter climate. In particular, it has shown us that a radiotelephone link between the site and the Snow Research Centre was not sufficiently reliable to be used in a real operational context. Tuning of a reliable, cold-resistant, self-contained power supply was also performed at this site. The signals recorded there, although we lack avalanche observations, extend our collection and are used as a blind set of signals. Cross-checking of the date and time information of the signals recorded at La Lavey and Saint-Christophe allows us to identify an earthquake before we receive the earthquake events reported and published weekly by the Laboratoire de Géophysique (see section 1.2). The inhabited, easily accessible, betterequipped Saint-Christophe site has a more operational and immediate approach. The accounts of the residents and the avalanche detectors allow us to check the performance of our system soon after each period of avalanches and to make immediate modifications if necessary. The aim of maintaining two sites is to investigate the effects of the site surrounding the station on the quality of the recorded signals.

\subsection{Methodology for a posteriori identification of the signals}

These two experimental sites have been working satisfactorily since 1992 and have allowed us to record about 300 signals during each campaign. Some signals are actually avalanches, others are extraneous signals such as earthquakes, blasts, rolls of thunder, helicopter sounds or animal footsteps. Therefore, we have had to learn how to recognize avalanche signals. For this, we have developed a methodology of a posteriori identification of signals to obtain a reliable training set of signals of an unambiguous origin. The principle is to determine the origin of each signal according to additional information. Earthquakes can be recognized by comparing the date and time of the events to the date and time of earthquakes listed in the report published weekly by the Laboratoire de Géophysique. Data from the French Sismalp network have also been used for that purpose. Blast signals are also easy to recognize, because they produce short, easily recognizable signals. For truck or helicopter sounds, the problem is more complicated. Again, we try to obtain information from the local council, helicopter companies or residents to confirm whether a truck, snowplough or helicopter was present at a given date and time corresponding to a recorded signal. Thunder rolls are discriminated according to weather conditions and interrogation of local residents. Finally, avalanche signals can be identified from accounts of local residents, National Park wardens, skiers or climbers. At the Saint-Christophe site, many avalanche signals have been identified by using avalanche detectors described in the previous paragraph.

This methodology of a posteriori identification has been carried out for 3 years at two sites. A large collection of unambiguous signals has been recorded. We have noticed that only about $15 \%$ of the recorded signals are actually produced by avalanches, the others being produced by earthquakes, blasts, animals, as well as helicopter, vehicle or thunder sounds. In practice, it is therefore insufficient to record merely the seismic activity of the site to estimate its avalanche activity. We have had to find a reliable method to determine avalanche signals automatically without additional information.

\section{AUTOMATIC SYSTEM FOR AVALANCHE- SIGNAL RECOGNITION}

\subsection{Construction}

A detailed analysis of about 200 unambigous signals recorded at the Saint-Christophe site in 1992, 1993 and 1994 has been carried out. This can be subdivided into 
three domains: time (envelope-shape analysis), timefrequency (evolution of the dominant frequencies vs time using the ARCAP method) and polarization (localization of the linear motions in a time-frequency plane). From all these preliminary studies, avalanche signals showed no discriminant particularity. Only global trends could be derived: avalanches generally give long jerky signals, whose frequency tends to increase as the avalanche moves towards the sensor. However, there are many exceptions to that rule, though many other types of non-avalanche signal can well be recognized on their envelope shape or their frequency content, or their polarization behaviour. After several years of study of various types of signal, an elimination process has therefore been adopted: to know whether a given signal has been produced by an avalanche or not, a whole group of tests (envelope shape, frequency content, etc.) has been implemented to determine whether it could be one of the well-known nonavalanche signals. If all the tests fail, then the signal is likely to be an avalanche. All the methods have first been programmed and tested using the MUSTIG interactive signal-analysis software developed at the Centre d'Étude des Phénomènes Aléatoires Géophysiques Research Centre on Random Geophysical Phenomena) in Grenoble. Then, the selected methods have been rewritten in $\mathrm{C}$ on the IBM RISC 6000 calculator of the CEN and implemented on the PC set up at the Saint-Christophe site.

\subsection{Structure}

The structure of the analysis and decision system is shown in Figure 3. It consists of three separate programmes. As this paper is not concerned with signal processing but rather with the operational applications of the seismic detection of avalanches, the methods used and the detailed structure of each programme will not be discussed here. The technical aspect of the system has been fully described in a paper submitted in June 1996 by B. Leprettre, N. Martin, F. Glangeaud and J. P. Navarre to IEEE Transactions on Signal Processing.

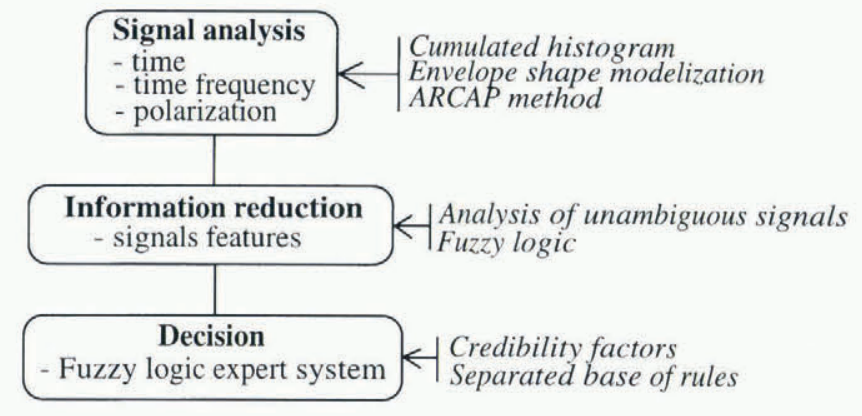

\section{Fig. 3. Flow charl of the SARA automatic analysis/ decision system for avalanche recognition.}

The first programme, which receives the threecomponent seismic signal, performs the time-frequencypolarization analysis. The ARCAP method (Dubesset and others, 1987) is used for time-frequency analysis. The polarization analysis is performed by estimating a linear polarization criterion after Capon frequency-filtering (Capon, 1969). The programme creates a file summarizing the characteristics of the signal in each domain: envelope-shape parameters, evolution vs time of the main frequency components, date and frequency of the linear motions.

This file is the input of a second programme which evaluates 16 pertinent signal features (or decision criteria). These descriptors are derived from the analysis results and fuzzy sets (Zadeh, 1965) which have been set up according to the empirical knowledge obtained from the analysis of the well-known signals. Each feature represents a pertinent characteristic of the signal that helps to determine its origin. It is given a value ranging from 0 (totally false) to 1 (totally true) representing its truth value, i.e. to what extent that pertinent characteristic is true of that particular signal. The three analysis domains are involved in the estimation of the signal features, which are given below:

\section{Time domain}

The signal is long; the signal is short; the average amplitude is low; the envelope shape is (is not) like an earthquake; the envelope shape is (is not) like that of an avalanche.

\section{Time-frequency domain}

Only high-frequency content; only low-frequency content; no high-frequency component; one frequency component is low; one component has a typical helicopter value; broadband frequency content; the dominant time frequency component increases (decreases).

\section{Polarization domain}

The signal onset zone is polarized; typical earthquake polarization pattern; a high proportion of linear waves come from the road.

Finally, the truth values of those features are the input of the last programme, which combines these pertinent criteria according to empirical rules and produces a diagnosis of the signal origin. The uncertain nature of the rules on which the diagnosis is based, induced by the fact that they have been established empirically from the analysis of well-known signals, is taken into account using credibility factors (Shortliffe and Buchanan, 1975). These are numbers ranging from 0 (a totally unreliable rule) to 1 (a totally reliable rule), representing what confidence one has in a given rule. The rules, and the inference system, have been programmed in such a way that they are easily identifiable, and that the system is able to explain its reasoning. The output of this third module is the truth value of all the possible conclusions concerning the signal origin, i.e. how well the signal is related to either a well-known signal in terms of envelope shape and time-frequency polarization behaviour. This enables us to decide whether the signal has been produced by an avalanche or not, and to estimate the reliability of this decision.

Figure 4 shows an example of signal analysis provided by the system for a "typical" avalanche signal. Figure $4 \mathrm{a}$ is the three-component seismic signal, Figure $4 \mathrm{~b}$ is the ARCAP time-frequency analysis of the vertical component, summarizing visually the output of the analysis module. Figure $4 \mathrm{c}$ is a list of signal features and the 


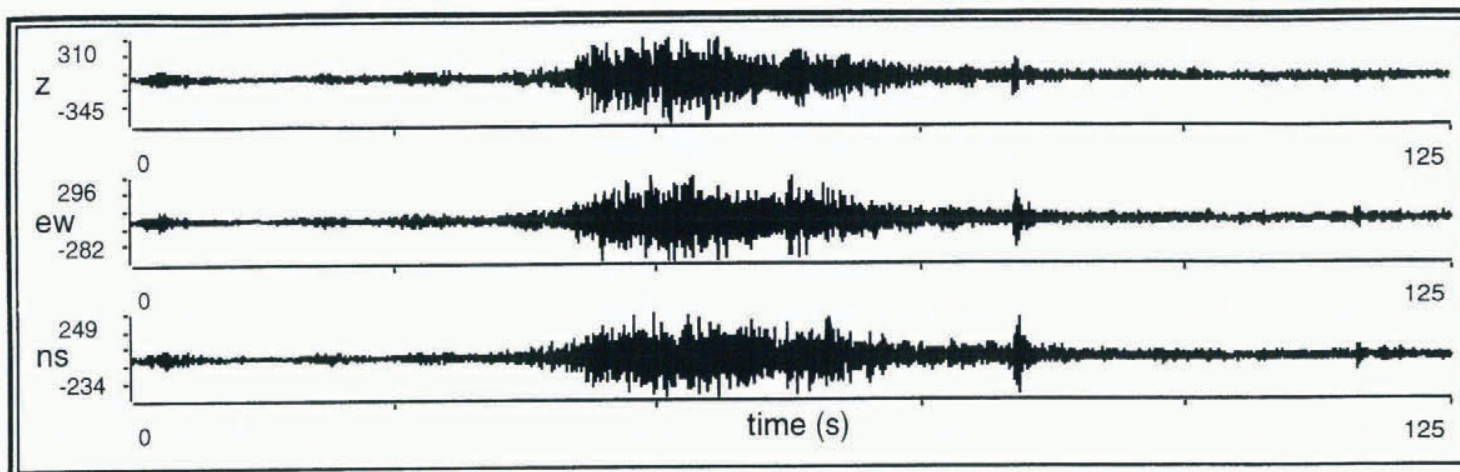

(a)

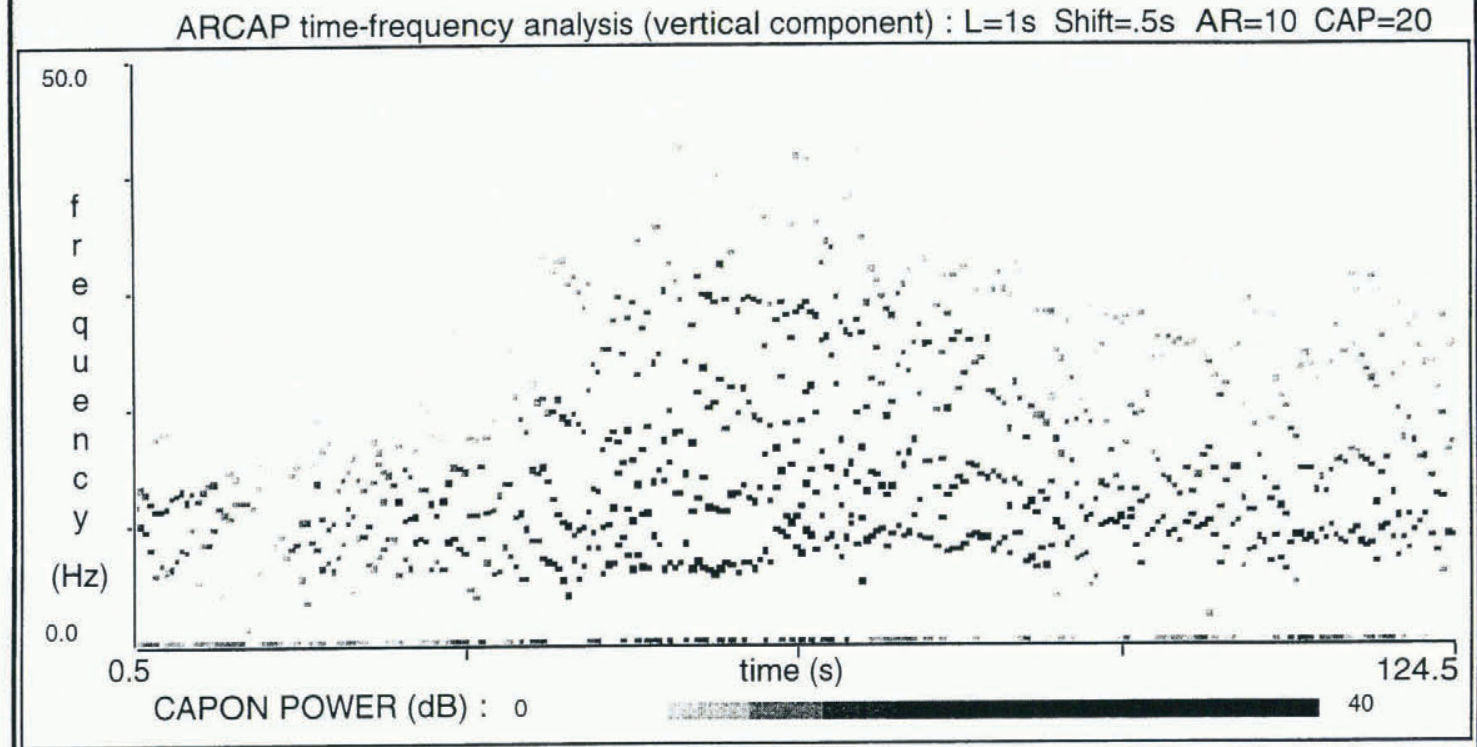

(b)

\section{SIGNAL DESCRIPTORS}

$\begin{array}{ll}\text { Short signal } & : 0.0 \\ \text { Long signal } & : 0.0 \\ \text { Average amplitude is low } & : 0.14 \\ \text { Earthquake shape } & : 0.49 \\ \text { Avalanche shape } & : 0.62 \\ \text { Only high frequency content }: 0.0 \\ \text { Only low frequency content }: 0.0 \\ \text { No high freq. component } & : 0.89 \\ \text { One component is under 5Hz } & : 0.0 \\ \text { Helicopter caract. freqency : } 0.0 \\ \text { Broadband frequency content }: 0.0 \\ \text { Dominant freq. increases } & : 0.50 \\ \text { Dominant freq. decreases } & : 0.0 \\ \text { Signal onset is polarized } & : 0.0 \\ \text { Earthquake polar. pattern } & : 0.0 \\ \text { Linear waves come from road : } 0.0 & \end{array}$

(c)

Fig. 4. Output of the system for a well-defined avalanche signal. (a) Three-component temporal signal; (b) ARCAP time-frequency analysis of the vertical component $z$, showing the power of the signal as a function of time and frequency; (c) Signal features derived from the analysis by the second module; (d) Conclusion provided by the third module from the signal features.

associated truth values (output of the second module). Finally, Figure 4d shows the diagnosis made by the third module. As this signal has a satisfactory signal-to-noise ratio and a somewhat typical avalanche behaviour in terms of temporal shape and time-frequency evolution, no diagnosis other than "avalanche" has been found by the decision module. That is to say, this signal is not like any non-avalanche signal we know, the only possible conclusion is therefore "avalanche".

On the other hand, Figure 5 shows another example of signal analysis where two different conclusions are provided by the expert system. The signal is actually an earthquake. The temporal aspect of the signal is not much different from some avalanche signals, so that a time- 


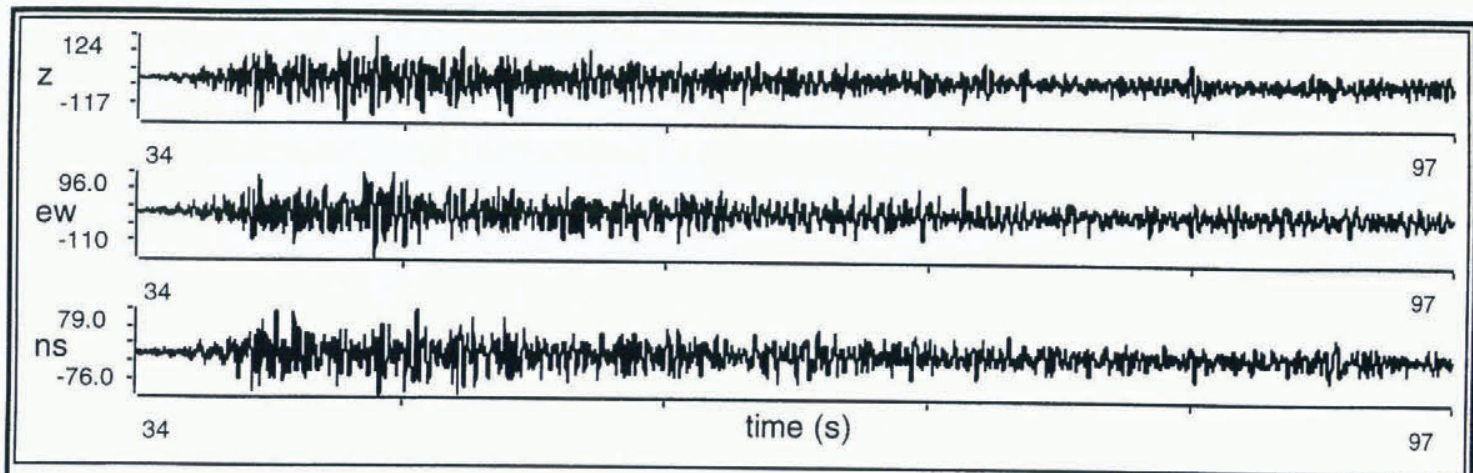

(a)

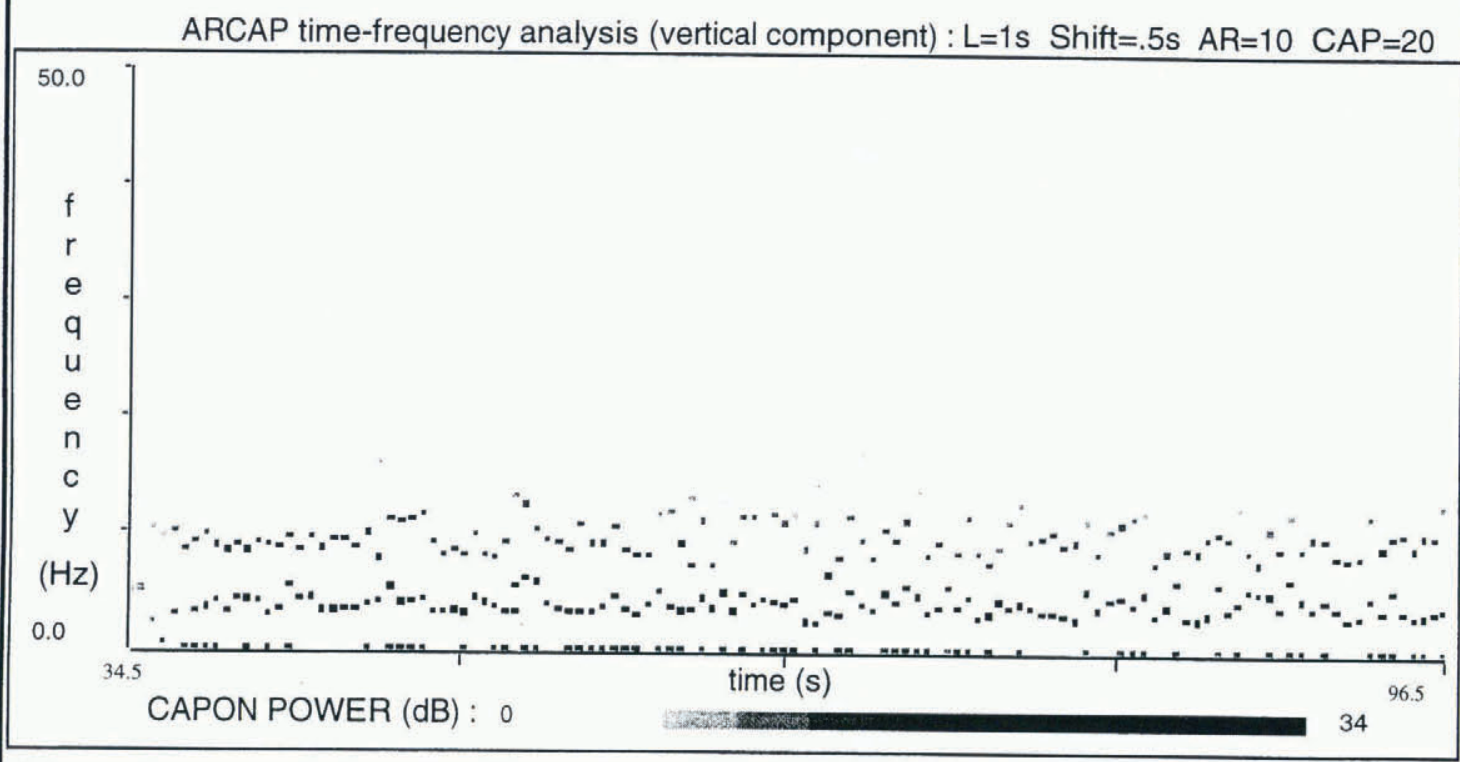

(b)

SIGNAL DESCRIPTORS

\begin{tabular}{|c|c|c|c|}
\hline $\begin{array}{l}\text { Short signal } \\
\text { Long signal } \\
\text { Average amplitude is low } \\
\text { Earthquake shape } \\
\text { Avalanche shape } \\
\text { Only high frequency content } \\
\text { Only low frequency content } \\
\text { No high freq. component } \\
\text { One component is under } 5 \mathrm{~Hz} \\
\text { Helicopter caract. freqency } \\
\text { Broadband frequency content } \\
\text { Dominant freq. increases }\end{array}$ & $\begin{array}{ll}: & 0.0 \\
: & 0.0 \\
: & 1.0 \\
: & 0.0 \\
: & 0.95 \\
: & 0.0 \\
: & 0.0 \\
: & 1.0 \\
: & 1.0 \\
: & 0.0 \\
: & 0.0 \\
: & 0.25\end{array}$ & & CONCLUSIONS \\
\hline $\begin{array}{l}\text { Dominant freq. decreases } \\
\text { Signal onset is polarized } \\
\text { Earthquake polar. pattern } \\
\text { Linear waves come from road }\end{array}$ & $\begin{array}{ll}: & 0.25 \\
: & 0.50 \\
: & 0.0 \\
: & 0.0\end{array}$ & (1) & $\begin{array}{l}\text { Earthquake: } 908 \\
\text { Avalanche : } 40 \%\end{array}$ \\
\hline
\end{tabular}

(c)

Fig. 5. Output of the system for a non-typical mid-range earthquake. (a) Three-component temporal signal; (b) ARCAP time-frequency analysis of the vertical component $z ;(c)$ Signal features derived from the analysis by the second module; (d) Conclusions provided by the third module from the signal features. For this signal, two conclusions are considered: the signal is well recognized as an earthquake (90\%) but it is also found to be behaving like an avalanche at a lower limit $(40 \%)$.

frequency analysis is necessary to reach a conclusion. From both temporal shape and evolution of the main frequency component, our system concludes that the avalanche hypothesis can be considered $40 \%$ true. But, given the low magnitude of the signal and the rather low $(<8 \mathrm{~Hz})$ frequency content and the absence of significant high $(>13 \mathrm{~Hz})$ frequency waves, it also concludes that the earthquake hypothesis is $90 \%$ true. In this case, several conclusions are proposed by the expert system. The signal characteristics are not sharp enough to lead to a single conclusion. In this particular case, the membership value of the signal in the "earthquake" class is $90 \%$ and only $40 \%$ in the "avalanche" class. Thus, the final conclusion here will be "earthquake". For a few signals, the system 
may propose three or four different conclusions, some of them having close membership values. In that case, one must admit that the signal origin cannot be clearly determined using only time, time-frequency and polarization information.

\subsection{Performance}

The system described in the previous paragraph, named SARA Système d'Analyse pour la Reconnaissance des Avalanches; analysis system for avalanche recognition), has been tested on a population of 294 signals of wellknown origin, including 13 avalanches. This test sample corresponds to events of unambiguous origin recorded in the Saint-Christophe site during the winter 1994 95. The avalanche origin of the 13 avalanche signals has been clearly established from observations and accounts of residents. All the signals of uncertain origin have been removed from this test set. The results of the automatic classification are the following:

\section{Success}

Twelve actual avalanche signals out of $13(92 \%)$ have been correctly classified into the AV class and 268 nonavalanche signals out of $281(95 \%)$ have been correctly rejected into the NAV class. Thus, 280 signals out of 294 $(95 \%)$ have been correctly classified. Blasts, teleseismic events and helicopter sounds are all classified in the NAV class.

\section{Failure}

One avalanche signal out of $13(8 \%)$ has been erroneously classified into the NAV class, due to the high proportion of linear waves coming from the road, leading to the "vehicle" diagnosis. Thirteen nonavalanche signals out of $281(5 \%)$ have been considered as avalanches. Among them, 12 are non-teleseismic earthquakes and one is a roll of thunder. There were 69 non-teleseismic earthquake signals and 35 thunder signals in the test sample.

The unsatisfactory success rate for the local earthquakes, although unpleasant for the mind, is not too prejudicial in an operational context. During an avalanche episode, lasting typically 2 or $3 \mathrm{~d}$, a maximum of one or two local earthquake events are statistically likely to occur. Thus, even if they are both erroneously classified as avalanches, they will not perturb the results too much, as many (typically 5-15) actual avalanche events will have occurred within the same period of time. In practice, the effect of this bad result concerning earthquake rejection is that, from time to time, a single avalanche is signalled even though the avalanche activity is actually low. Further studies are in progress and we expect to improve in the near future the rejection of earthquakes using a single station.

In the test sample, the avalanche signals represent only about $5 \%$ of the global population. We do not have a large collection of unambiguously identified avalanche events. It is therefore difficult for the moment to conclude that the average correct recognition rate will still be about $90 \%$ with another set of avalanche signals. Many other signals have been classified as avalanches (see the results for January and February 1995 in section 3) but could not be unambiguously identified as avalanches, although most of them are not earthquakes, mining blasts or claps of thunder. Hence, these signals could not be included in the test sample we used to measure the performance of our system. Nevertheless, in the next part of this paper we compare the results of our system with a set of data related to avalanche activity. This can be considered as another kind of check to estimate the quality of recognizing avalanche signals.

\section{OPERATIONAL RESULTS}

The SARA system for the automatic recognition of avalanche signals has been working satisfactorily in preoperational conditions since 5 January 1995 at the SaintChristophe site. The recorded signals are analysed every $24 \mathrm{~h}$. For each event the following data are transmitted to the laboratory: date and time of occurrence, conclusion provided by the expert system and associated truth value. The number of signals detected as avalanches gives an estimate of what is called the seismic avalanche activity. In order to estimate the consistency of the results provided by SARA and evaluate the potential contribution of seismic detection to avalanche science, the results of the SARA system are compared with other data related to avalanche activity. Two main aspects will be successively examined: estimation of the daily avalanche activity and real-time surveillance of avalanche-prone areas.

\subsection{Comparison between the daily seismic aval- anche activity and other data}

To estimate the quality of SARA's results, the daily seismic avalanche activity can be compared with data quantifying the avalanche activity or the risk of avalanche. The first piece of data is the number of avalanches observed each day by the ski patrols of the Les Deux-Alpes ski resort, close to the Saint-Christophe site. The second is the degree of natural avalanche risk on the new European Avalanche Hazard Scale (EAHS). It ranges from 1 (low degree of risk) to 5 (very high degree of risk) and is estimated daily by the weather station at Saint-Martin-d'Hères. When the EAHS rating is larger than 2, one can assume that medium- or large-sized natural avalanches are likely to occur. Finally, the third factor is the average risk of avalanche provided by the SAFRAN-CROCUS-MEPRA expert system considering a southwest aspect slope with a slope of $40^{\circ}$, at an altitude of $1800-3000 \mathrm{~m}$ in the Oisans Massif, French Alps. It is based on a deterministic simulation of the snow-mantle evolution in each massif, given the altitude, aspect and steepness of the slope considered. Two estimates of avalanche risk are made each day: one at $0600 \mathrm{~h}$ UTC and one at $1200 \mathrm{~h}$ UTC.

The first two graphs in Figure 6 show the daily global seismic activity for January 1995 at the Saint-Christophe site (a) and the seismic avalanche activity after automatic sorting of the signals by the SARA system (b-black bars). It highlights the fact that it is necessary to analyse the recorded signals in order to determine whether they were produced by an avalanche. In fact, the seismic 
a General seismic activity—January 95

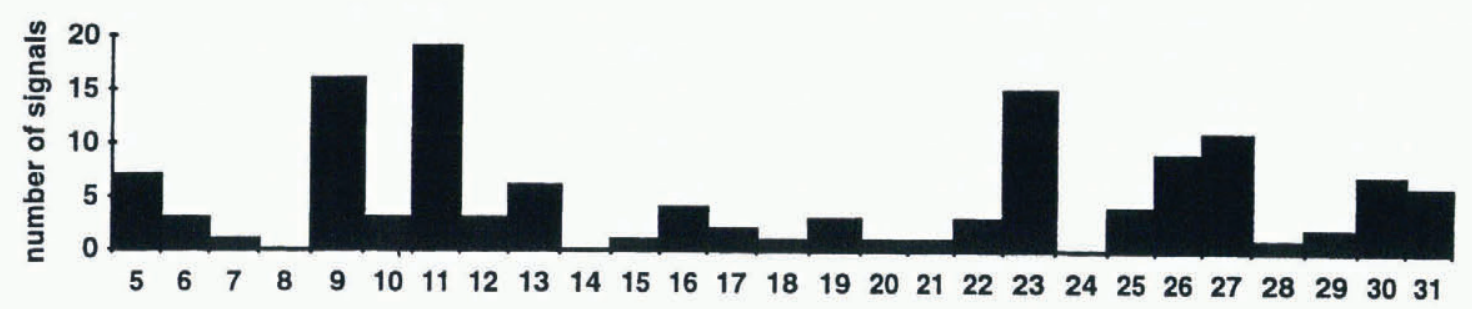

b Seismic avalanche activity compared with visual observations

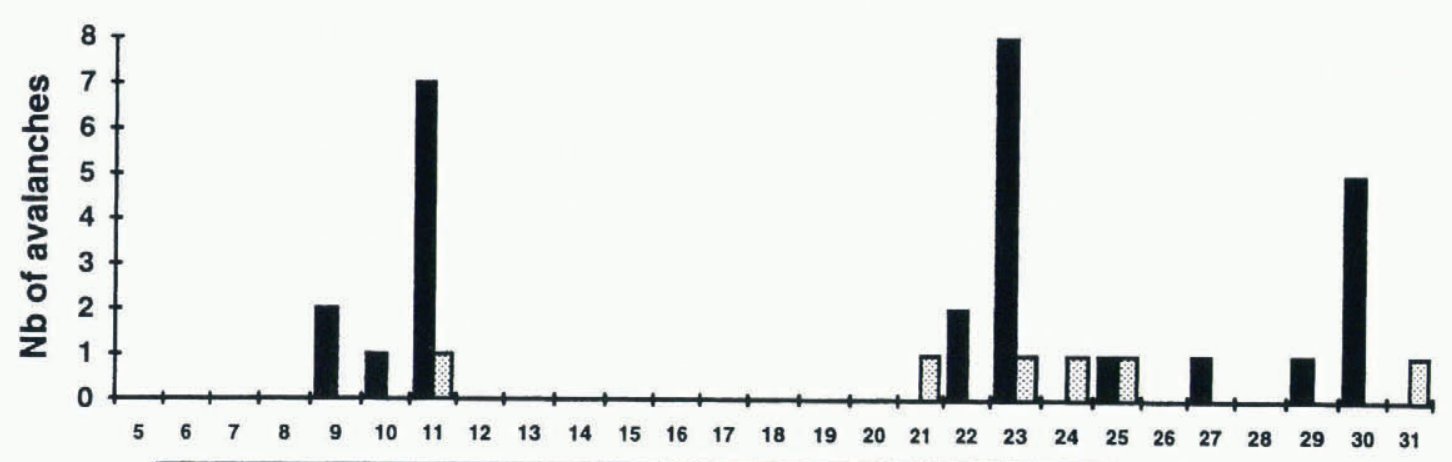

SARA seismic avalanche activity Deux-Alpes Observations

c Forecast degree of risk of natural avalanche (EAHS scale)

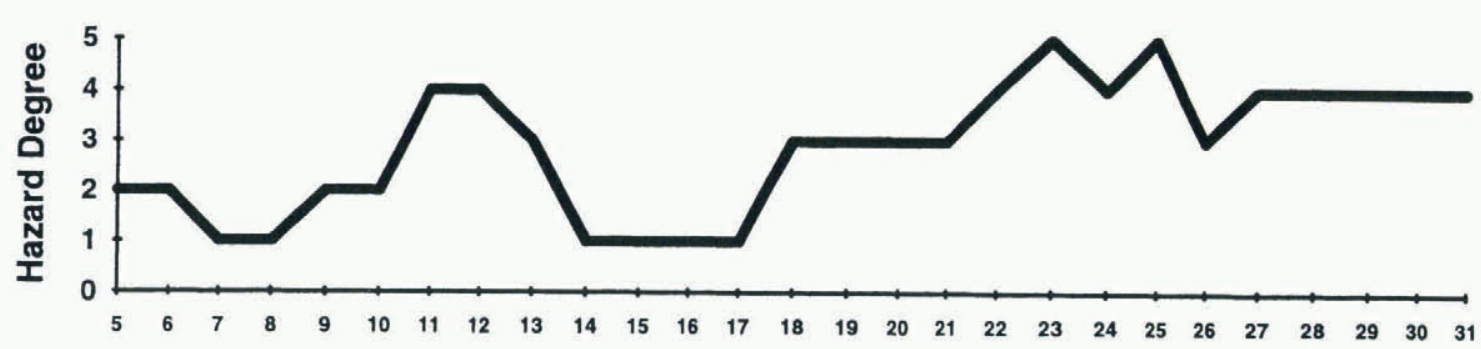

d Average natural degree of risk (MEPRA simulation)

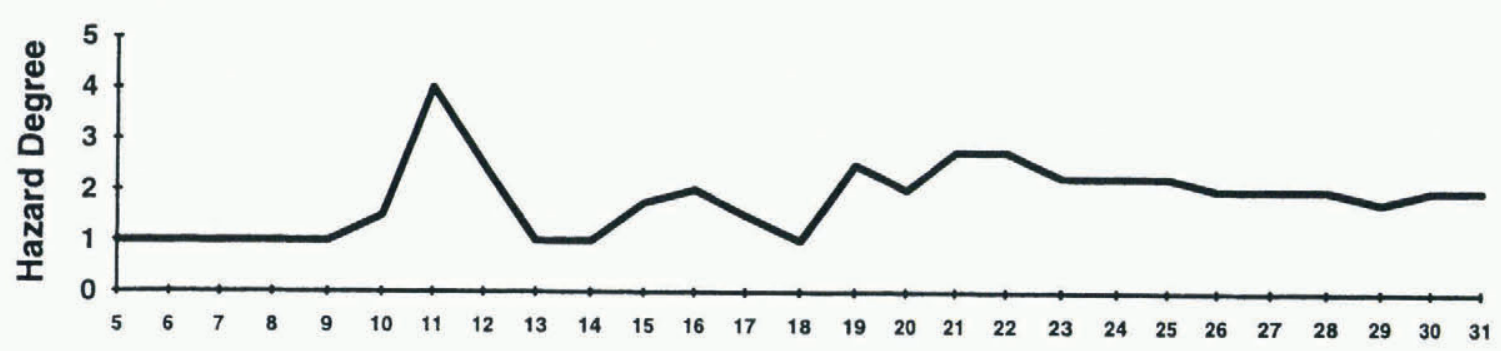

Fig. 6. Results provided by the SARA system for the period between 5 and 31 January 1995 compared with other dala. (a) Daily global seismic activity; (b) Seismic avalanche activity derived by the SARA system (black bars) and visual avalanche observations at the Les Deux-Alpes ski resort (grey bars); (c) Forecast natural avalanche-hazard rating; (d) Natural avalanche-hazard rating estimated by the SAFRAN-CROCUS-MEPRA chain. 
activity due to avalanches can be low (see 9 January), while at the same time the seismic activity in general can be high because of public works mining, or numerous helicopter flights due to mountain-rescue operations, or thunderstorms, etc. Using later information, it was established that the 5 January peak in Figure 6a was produced by numerous mining blasts. On 9 January, we were on site for a technical visit, causing many footstep signals. The general seismic activity peak for 27 January corresponds to a series of artificial avalanche releases at the Les Deux-Alpes ski resort. All these non-avalanche seismic activity episodes are well rejected by the SARA system. Among the 28 signals which have been classified as avalanches, three have been identified as actual avalanches. As for the 25 other signals, we cannot prove that they correspond to avalanches but the a posteriori identification process showed that none of them could be earthquakes or mining blasts. Their origin could not be clearly established. Given the properties of most of these signals (time, frequency), it seems reasonable to think that a large proportion of these signals have actually been produced by avalanches.

Figure $6 \mathrm{~b}$ also shows the number of avalanches reported daily by the Les Deux-Alpes ski resort (grey bars). The correlation between the seismic avalanche activity and the visual observations provided by the Les Deux-Alpes ski resort is small. Some periods (29 and 30 January, for example) with considerable avalanche conditions show no observations at all. Moreover, it proves impossible to estimate the intensity of a period of avalanches only using visual observations: few avalanches can be observed whatever the seismic avalanche activity or the EAHS degree. There are two reasons for this. First, visual observation of avalanches is impossible at night or when the weather is bad. This creates a time gap between the avalanche occurrence and its report. Secondly, these episodes correspond to fresh-snow airborne-powder avalanches. The deposits of such avalanches are sometimes almost invisible and therefore difficult to observe even when the weather is clear. This creates a second bias in terms of the intensity of the period of avalanches. It therefore appears that visual observations alone are not reliable sources for estimating the actual avalanche activity of a massif because the information is imprecise. On the other hand, the seismic avalanche activity varies from one to eight signals detected for each episode. It therefore seems more likely to provide reliable information: the time bias is eliminated as the system keeps track of the data and time of each avalanche occurrence, and the intensity bias seems smaller as the seismic avalanche activity varies significantly from one episode to another. Let us compare these results with other data.

The bottom part of Figure 6 shows, for the same period of time, the evolution of the natural avalanche degree of risk on the new European Avalanche Hazard Scale (EAHS) forecast by the weather station at SaintMartin-d'Hères (Fig. 6c) and the evolution of the risk of natural avalanche predicted by the deterministic expert system SAFRAN-CROCUS-MEPRA (Fig. 6d). As the figures provided by those sources have not exactly the same significance, it is out of the question to compare the numerical values for a given day. The point here is rather to compare the evolution vs time of those figures with the evolution of the seismic avalanche activity provided by the SARA system. There is a strong correlation between the seismic avalanche activity and both EAHS and MEPRA avalanche-risk estimates: the periods with intense seismic avalanche activity actually correspond to periods when the natural avalanche hazard is high or very high (10 and 11 January, for example). The beginning of the period of avalanches between 22 and 23 January is shown well. However, the SARA system shows an increase of avalanche activity on 30 January, while both MEPRA and the weather station find no increase in the hazard degree. (The EAHS hazard degree remains high within the whole period between 22 and 31 January but shows no increase on 30 January.

The same data are presented for February 1995 in Figure 7. Once again, the automatic system separates the actual avalanche signals, the so-called seismic avalanche activity (Fig. 7b; black bars), from the seismic activity in general (Fig. 7a). For example, the general seismic activity peak on 5 and 6 February was produced by one mining blast, one local earthquake and two helicopter signals, and is correctly rejected as non-avalanche. Among the 12 signals which are classified as avalanches, three have been identified as actual avalanches. The origin of the nine remaining signals could not be established as earthquakes or mining blasts or thunder. Once again, it seems reasonable to consider that a large proportion of these were actual avalanche signals. The correlation between the seismic avalanche activity and the observed avalanche activity (Fig. 7b; grey bars) is good for the period between 13 and 17 February (many avalanches have been observed) but poor for the period between 23 and 26 February (only one observed avalanche during this period). Figure $7 \mathrm{c}$ shows a good correlation between the predicted natural avalanchehazard rating and the seismic avalanche activity. The period between 23 and 26 February, during which only one avalanche has been visually observed, appears to be a very active avalanche episode (the EAHS hazard degree reaches its maximum value on 26 Feburary). The SARA seismic avalanche activity is actually high during this period, although a $24 \mathrm{~h}$ time shift appears with respect to the forecast EAHS ratings. This period is also well distinguished by MEPRA (Fig. 7d), the average hazard degree reaching 4 . However, the period between 13 and 17 February, when several avalanches were observed and detected by SARA, does not appear well. The reason for this could be that the MEPRA simulations are run at $0600 \mathrm{~h} \mathrm{UTC}$ and $1200 \mathrm{~h}$ UTC each day. In mid-February many avalanches are released in the late afternoon by heating and humidification of the snow mantle. The MEPRA simulation is therefore run too early to detect those conditions favourable to avalanches.

These results show that, with a few exceptions, it is generally impossible to derive a reliable estimate of avalanche activity solely from visual observations. The seismic detection of avalanches, coupled with an automatic avalanche-signal recognition system, seems to be able to quantify the intensity of an avalanche episode. This information could be useful for the short-term prediction of the risk of avalanches and for the improvement of our knowledge about the avalanche phenomenon. However, this is an experimental system 
a General seismic activity-February 95

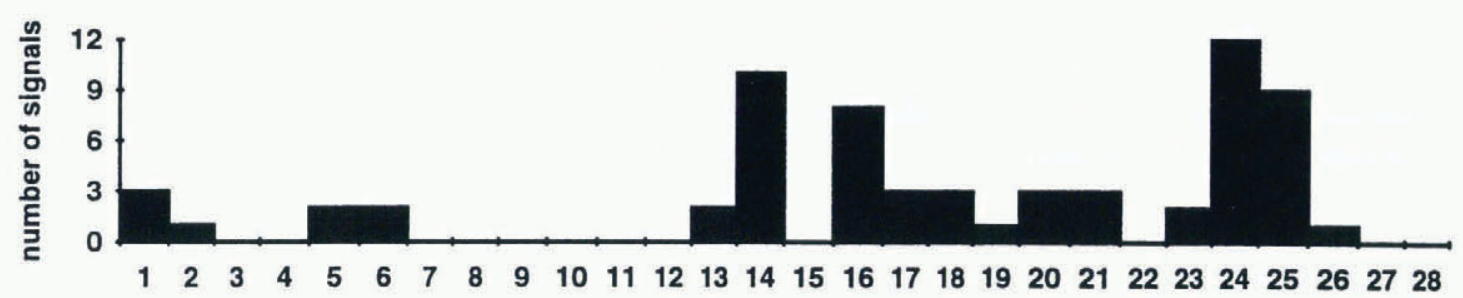

b Seismic avalanche activity compared with visual observations

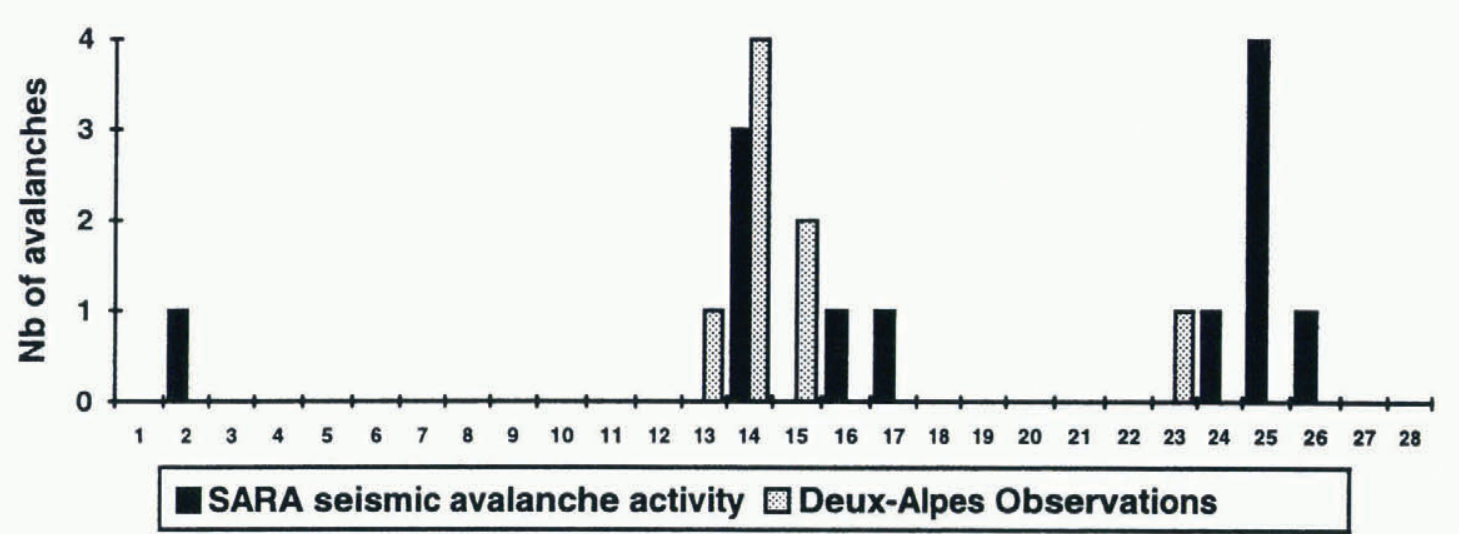

c Forecast natural degree of risk (EAHS scale)

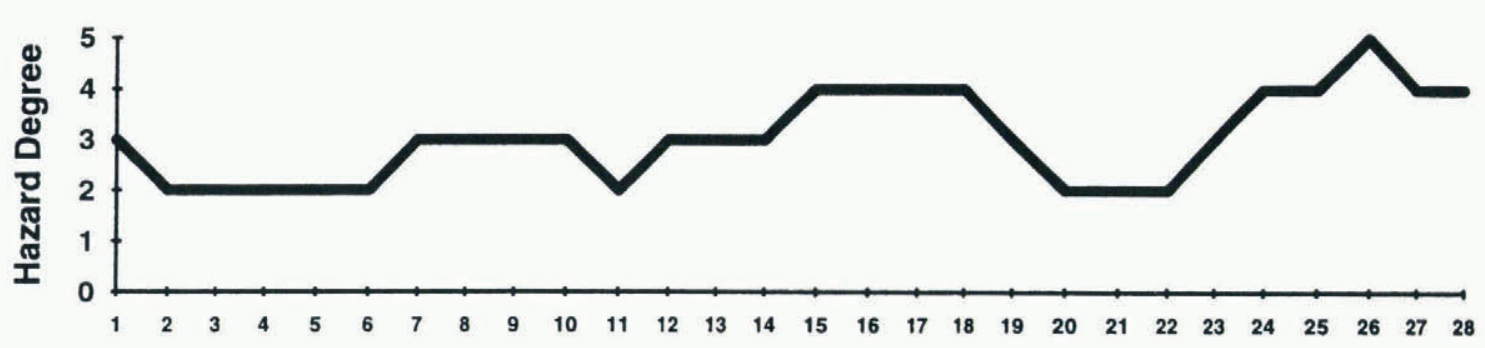

d Average natural degree of risk (MEPRA simulation)

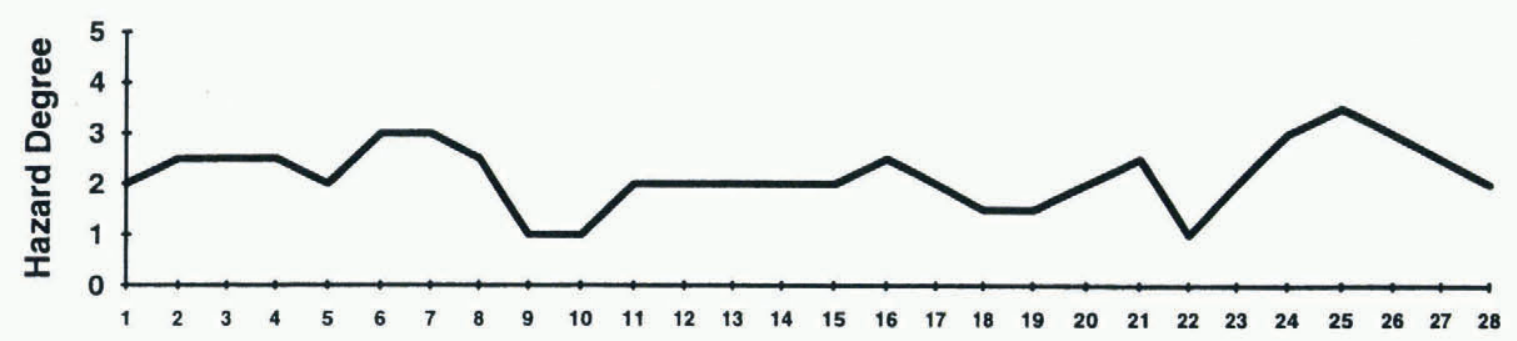

Fig. 7. Results provided by the SARA system for the period between 1 and 28 February 1995 compared with other data. (a) Daily global seismic activity; (b) Seismic avalanche activity derived by the SARA system (black bars) and visual avalanche observations at the Les Deux-Alpes ski resort (grey bars); (c) Forecast natural avalanche-hazard rating; (d) Natural avalanche-hazard rating estimated by the SAFRAN-CROCUS-MEPRA chain. 
and only one pre-operational station has been installed. We must therefore be cautious about these first results, even though they are extremely encouraging. This point will be further discussed in the concluding remarks.

\subsection{Semi-instantaneous surveillance of avalanche- prone zones using seismic detection}

Another use of seismic detection of avalanches is the almost immediate surveillance of avalanche-prone areas. Instead of being analysed only once a day, the signals can be analysed as soon as they have been recorded. This almost immediate avalanche detection, given the current and forecast weather conditions, makes it possible to forecast the short-term risk of avalanches and therefore facilitate decisions on closing roads or ski resorts. This has not yet been done in practice this year but a simulation was carried out between 10 and 12 January by plotting vs time every hour the number of signals recognized as avalanches by our system (see Fig. 8). In addition, we have shown on the same graph the total height of the snow layer recorded at the automatic "Nivose" weather station at Les Ecrins, whose height and position are similar to the Saint-Christophe site. It is clear from this figure that the avalanche period begins as soon as the thickness of the fresh-snow layer reaches about $35 \mathrm{~cm}$. When several avalanche signals are observed within a short time span and the snowfall is still continuing, one can decide to close exposed ski tracks or roads (here around $2100 \mathrm{~h}$ on 10 January). Significant avalanche activity is observed until the snowfall stops, then the natural avalanche activity soon ceases. The roads could have been opened, quite safely, round $1800 \mathrm{~h}$ on 11 January. These results confirm what avalanche forecasters empirically knew about the beginning of an avalanche episode: ski patrols usually start releasing avalanches with explosives as soon as the fresh-snow layer reaches 35 or $40 \mathrm{~cm}$. The end of the natural avalanche episode, on the other hand, seems to come sooner than previously thought. However, we must be cautious as this result has been obtained with one single, experimental SDA station. Further studies are necessary before we are able to draw reliable conclusions on forecasting the end of an avalanche period. It must be

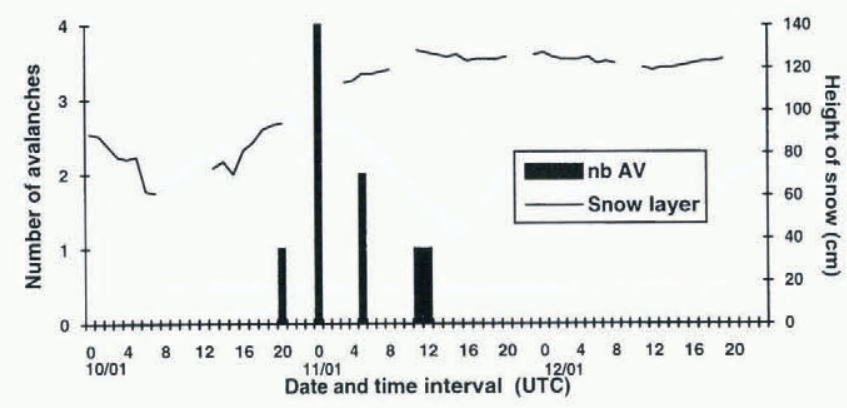

Fig. 8. Seismic avalanche activity during the period between 10 and 12 January 1995 with a 1 h time step. Number of signals delected and recognized as avalanches each hour (black bars) and total height of snow layer at the Les Ecrins automatic nivo-meteorological station (black line). noted that, for the time being, the seismic detection of avalanches cannot be used in avalanche-alarm systems: the signal-recognition process is carried out using the whole signal, once it has been recorded, which is too late to give the alarm. The only solution would be to take a quick decision when faced with only the very beginning of a signal, which would not be reliable, given the difficulty of recognizing an avalanche. Still, these results show the interest of the seismic detection of avalanches for the almost immediate surveillance of avalanche-prone zones and the study of avalanche-activity evolution with a high time resolution.

\section{GONCLUDING REMARKS}

The seismic detection of avalanche (SDA) is able to produce a reliable, objective, almost immediate estimate of avalanche activity on a massif. Although there is still room for improvement, the initial research into an automatic system for recognizing avalanche signals developed at the Centre d'Étude de la Neige gives encouraging results. During the winter of 1995, avalanche forecasters at the Saint-Martin-d'Hères meteorological station regularly asked for recent SDA data to confirm their forecasts or to help their evaluation of current avalanche activity.

The range of the SDA system is difficult to evaluate, as the triggering of the detection device and the signal-tonoise ratio depend on several factors: type, quantity and speed of the moving snow, sliding-surface characteristics, configuration of the avalanche path and, of course, distance between the sensor and the avalanche. Some small loose-snow avalanches $200 \mathrm{~m}$ away from the sensor have not been detected, while large airborne-powder avalanches have been recorded up to $10 \mathrm{~km}$ away from the sensor. However, we can estimate that the effective range, that is to say the distance from which "common" avalanches can be detected with a satisfactory signal-tonoise ratio, is about $46 \mathrm{~km}$. As many slow, loose-snow avalanches occur in spring, one can expect our system to be less efficient in spring than in winter, when dynamic, airborne-powder avalanches are more likely.

Therefore, the equipped site must be well representative of a massif if one wishes to obtain a reliable estimate of avalanche activity inside the whole massif. At the same time, a good site must be seismically quiet (low background noise), close to avalanche corridors and suitable for radiotelephone or satellite transmission. The influence of the site and its representativeness will be more precisely investigated in the future. For example, a given site might record typically ten signals per day during an avalanche period, while at the same time another site might typically record only five signals per day. It is clear that some sites are more suitable than others, depending on the massif, the aspect or the number and configuration of the avalanche corridors within the effective range. The presence of rock faces or rocky avalanche corridors acts as a natural amplifier that improves reception. It could therefore be appropriate to take into account the suitability of each site when calculating the results.

From a practical point of view, an operational, selfcontrolling prototype is to be designed and tested during 
the winter of 1996. The analysis/decision software will be implemented on a PC card inside the seismic log, so that the whole SDA system will be compact. The analysis will be undertaken on site and the results will be transmitted to the Centre d'Étude de la Neige via a Météosat satellite link. The results will be instantly at the disposal of avalanche forecasters, so that the value of seismic detection of avalanches in a real operation context can be evaluated.

Now that most spurious signals are correctly rejected by the SARA system, tests should be carried out in skiing areas to determine whether the seismic signals generated by ski lifts, tamping machines or snow-makers are also satisfactorily rejected. If so, a SDA station could be installed in a skiing area where good daily avalanche surveys are carried out, in order to improve the comparison between seismic avalanche activity and visual observations.

At the same time, further studies will be undertaken on unambiguous signals. We hope to find new criteria to improve the recognition of non-avalanche signals, particularly earthquake signals. If this proves unsatisfactory, we could eventually solve this problem by using data from two distant seismic stations: if the date and time of an event are almost the same at two distant stations (given the distance between the stations and the average speed of seismic body waves), then the signal is surely an earthquake, because an avalanche could not have been picked up by both stations.

The problem of avalanche localization will also be further investigated: is it possible, with a single threecomponent sensor, to obtain a reliable estimate of the azimuth of the avalanche by observing the linearly polarized waves? This point is very important. The SARA system is not intended to become widely used, because of its cost: about 150000 French francs (US\$ 35000 ) for a seismic station including a hard disk for the storage of events and a PC for signal processing, plus a reliable power supply and a Météosat communication device. Therefore, it is out of the question to set up a network of seismic stations in a massif for locating avalanches. To take a long-term view, few massifs could be equipped with one SDA station. It could therefore be very interesting to estimate the azimuth of avalanche events with a single station. Given the relatively low range of this system, this could give a rough estimate of the geographical location of avalanches.

Finally, we will try to determine whether a given avalanche corridor has a proper "seismic signature" (depending on its topography) that could help both identification and localization of avalanche events.
Results from all these developments will be submitted later.

\section{ACKNOWLEDGEMENTS}

Y. Danielou (CEN) dealt with transmitting and filing the recorded signals. G. Giraud (CEN) supplied the outputs of the MEPRA expert system. J. Villecrose, an avalanche forecaster for the Centre Départemental Météorologique in Saint-Martin-d'Hères, provided the forecast naturalavalanche ratings.

N. Martin and F. Glangeaud, both with the Centre d'Étude des Phénomènes Aléatoires Géophysiques Research Centre on Geophysical Random Phenomena) in Grenoble, helped to select and develop the signalprocessing methods used in the analysis module.

Last, but not least, the residents of the Vénéon Valley and Saint-Christophe-en-Oisans, by kindly reporting all year round on the events they observed, allowed us to identify many avalanches or helicopter or vehicle signals.

\section{REFERENCES}

Capon, J. 1969. High resolution frequency wavenumber spectrum analysis. Proc. IEEE, 57, 1408-1418.

Dubesset, M., B. Berriani, J. L. Lacoume, N. Martin and C. Cliet. 1987. Analysis and modeling of seismic signals over short time windows. In Extended abstracts/Proceedings, 57th International Meeting, SEG, 11-15 October 1987, New Orleans. Society of Exploration Geophysics.

Giraud, G., E. Brun, Y. Durand and E. Martin. 1994. Validation of objective models to simulate snow cover stratigraphy and avalanche risks. In Proceedings, International Snow Science Workshop, 30 October-3 November 1994, Snowbird, Ulah, 509-517

Navarre, J. P., Y. Danielou, J. M. Panel, A. Taillefer and B. Lesaffre. 1991. Détection sismique des avalanches. In Actes du Symposium. ANENA-CISA-IKAR, 48 juin 1991, Chamonix, France. Grenoble, Association Nationale pour l'Étude de la Neige et des Avalanches, $148-153$.

Sabot, F., P. Martinez, E. Suriñach, C. Olivera and J. Gavaldà. 1995. Détection sismique appliquée à la caractérisation des avalanches. In Sivardière, F., ed. Les apports de la recherche scientifique à la sécurité neige, glace et avalanche/The contribution of scientific research to safety with snow, ice and avalanche. Actes de Colloque, Chamonix, 30 mai-3 juin 1995. Grenoble, Association Nationale pour l'Étude de la Neige et des Avalanches (ANENA), 19-24.

St. Lawrence, W. and T. R. Williams. 1976. Seismic signals associated with avalanches. 7. Glaciol., 17 (77), 521-526.

Shortliffe, E. H. and B. G. Buchanan. 1975. A model of inexact reasoning in medicine. Math. Biosci., 23, 351-379.

Valla, F. and J. L. Tuaillon. 1991. Accidents d'avalanches en France, statistiques des années 1970-1990. In Actes du Symposium. A.NENACISA IKAR, 48 juin 1991, Chamonix, France. Grenoble, Association Nationale pour l'Étude de la Neige et des Avalanches, 8-16.

Zadeh, L. A. 1965. Fuzzy sets. Inf. Control, 8, 338-353. 